\title{
In vitro and in vivo examination of anticolonization of pathogens by Lactobacillus paracasei FJ861111.1
}

\author{
Kan Deng, ${ }^{* 1}$ Tingtao Chen, ${ }^{11,2}$ Qinglong Wu,† Hongbo Xin, ${ }^{*}$ Qiang Wei, ${ }^{*}$ Ping Hu, ${ }^{*}$ Xiaolei Wang, ${ }^{*}$ Xin Wang, ${ }^{*}$ \\ Hua Wei, $\ddagger$ and Nagendra P. Shah $\dagger^{2}$ \\ *Institute of Translational Medicine, Nanchang University, Nanchang, Jiangxi 330031, China \\ †Food and Nutritional Science, School of Biological Sciences, The University of Hong Kong, Hong Kong \\ $\ddagger$ State Key Laboratory of Food Science and Technology, Nanchang University, Nanchang, Jiangxi 330047, China
}

\section{ABSTRACT}

Very limited information exists on the exclusion of pathogens by probiotics in the gut of the host challenged with pathogens. In this study, we tested probiotic characteristics in vitro and anticolonization ability of Lactobacillus paracasei FJ861111.1 in mice infected with selected pathogenic microorganisms. The in vitro results indicated that L. paracasei FJ861111.1 had a high survival in acidic conditions at $\mathrm{pH} 2.5$ and bile salt concentration at $0.3 \%$, and strong inhibition ability against common pathogens including Shigella dysenteriae, Staphylococcus aureus, Cronobacter sakazakii, Escherichia coli, and Candida albicans. The cell adhesion assays showed that L. paracasei FJ861111.1 exhibited strong adherence to HT-29 cells and excluded the adhesion of selected food-borne pathogens to HT29 cells. The in vivo results showed that fermented milk with L. paracasei and viili (a Nordic yogurt product) significantly improved the population of total bacteria and of Lactobacillus in the feces of mice, and significantly inhibited the colonization of $C$. albicans to the intestines of mice post- $C$. albicans infection. Thus, it appears that this strain could be used as a probiotic organism for manufacturing functional fermented milk. Key words: Lactobacillus paracasei, probiotic characteristics, anticolonization, pathogenic infection

\section{INTRODUCTION}

Consumption of fermented milks has shown several health-promoting benefits, including modulation of gut

Received April 28, 2015

Accepted June 20, 2015.

${ }^{1}$ These authors contributed equally to this work.

${ }^{2}$ Corresponding authors: chentingtao1984@163.com and npshah@ hku.hk microbiota, lowering of blood pressure, and reduction in the risks of bladder cancer and colon cancer. Fermented milks have a long history of consumption by humans (Alatossava et al., 2013). Viili, a semi-solid yogurt-type product originating from the Nordic countries, has a gelatinous consistency and a sour taste due to microbial action of yeasts and lactic acid bacteria. The product has been popular for its various biofunctionalities, such as reducing blood cholesterol level, preventing carcinogens, and enhancing host immunity (Kitazawa et al., 1991; Nakajima et al., 1992; Wang et al., 2008; Chen et al., 2013, Wu et al., 2013). Among the microbial consortium, lactic acid bacteria played an important role in the process of viili fermentation in regard to milk acidification, texture, flavor, and aroma development.

Probiotics are defined as "live microorganisms which, when administered in adequate amounts, confer a health benefit on the host" (Diplock et al., 1999; Hill et al., 2014), and lactic acid bacteria strains with beneficial properties have been increasingly used as probiotic starters for food fermentations for enhancing immune function and preventing gastrointestinal infections (Wood, 1998; de Roock et al., 2010; Higgins et al., 2010). Hence, candidate strains with probiotic features are very promising for industrial milk fermentation, such as manufacturing value-added fermented milks (Botta et al., 2014). To deliver its beneficial effects in the intestine, probiotics must be capable of surviving in the gastrointestinal tract, including extreme acidic environment in the stomach and bile salt conditions in the small intestine, and adhering to intestinal epithelial cells (Chae et al., 2013; Lamari et al., 2014, Ranadheera et al., 2014). Moreover, probiotics have been shown to bring a positive balance in the gut microbiota after an imbalance caused by antibiotic treatment or infections due to pathogens and other stress (Zhao, 2010; Zhang et al., 2012). This involves nutrient absorption, immune response, and colonization resistance to pathogens (Belkaid and Hand, 2014; Chen et al., 2014a; Fung et al., 2014). 
However, very limited clinical intervention or practice to overcome such imbalance has been carried out, especially for restoring the microbiota after infections with pathogens. Recently, probiotics have been suggested for modulating the microbiota; however, no guidance for choosing probiotics exists because of their strainspecificity. Lactobacillus paracasei FJ861111.1 was previously isolated from viili and exhibited good properties for milk curding and production of exopolysaccharides (Chen et al., 2013). In the present study, this strain was used to evaluate its acid and bile salt tolerance, antibacterial activities, inhibition of food-borne pathogens adhesion to HT-29 cells, and anticolonization effect in the gut of the mice post-Candida albicans infection. Another human-derived probiotic organism, Bifidobacterium longum ATCC15708, was included as a control due to its wide applications in food fermentation and as a probiotic organism (Gomes and Malcata, 1999).

\section{MATERIALS AND METHODS}

\section{Bacterial Strains and Cultivation Conditions}

Lactobacillus paracasei FJ861111.1 was previously isolated from viili (Chen et al., 2011a). In addition, $B$. longum ATCC15708 was used as a positive probiotic control in our study. Bifidobacterium longum and $L$. paracasei were cultivated in de Man-Rogosa-Sharpe (MRS) medium and incubated at $37^{\circ} \mathrm{C}$ for $24 \mathrm{~h}$ under anaerobic conditions. Pathogenic microorganisms including Shigella dysenteriae 301 and 2457, Staphylococcus aureus Cowan1 and CMC, Cronobacter sakazakii 45401 and 45402, Escherichia coli 44102, and C. albicans SC5314 were cultivated in Luria-Bertani (LB) medium and incubated at $37^{\circ} \mathrm{C}$ for $24 \mathrm{~h}$ under aerobic conditions. Organisms were propagated in corresponding media 3 times before making fermented milks and conducting experiments.

\section{Acid and Bile Salts Tolerance Assay}

Stock cultures of L. paracasei and B. Longum were propagated twice in MRS medium at $37^{\circ} \mathrm{C}$ for $24 \mathrm{~h}$ before the following assay. For the acid tolerance assay, the cultures were harvested by centrifugation at 4,500 $\times$ $g$ and $4^{\circ} \mathrm{C}$ for $10 \mathrm{~min}$, and bacterial cell pellet was then resuspended in PBS. The cell suspension was 100-fold diluted in PBS at various $\mathrm{pH}$ values, including1.5, 2.5, $3.5,4.5$, and 7.0 , and incubated at $4^{\circ} \mathrm{C}$ for $4 \mathrm{~h}$. For the bile salt tolerance test, fresh cultures were inoculated into MRS medium containing 0.1 to $0.5 \%$ (wt/wt) bile salts and incubated at $37^{\circ} \mathrm{C}$ for $4 \mathrm{~h}$. After incubation, the viable cells of $L$. paracasei and B. longum were examined using plate count method (Chen et al., 2014b).

\section{Antimicrobial Activity of L. paracasei and B. longum Agar Diffusion Assay}

The agar diffusion assay was carried out to examine the antimicrobial activity of $L$. paracase $i$ and B. longum as previously described (Chen et al., 2014b). Cultures of L. paracasei and B. longum grown anaerobically for $24 \mathrm{~h}$ in MRS medium were centrifuged to obtain supernatants. Overnight $(12 \mathrm{~h})$ cultures of the pathogenic microorganisms including Sh. dysenteriae 301 and 2457 , Staph. aureus Cowan1 and CMC, Cr. sakazakii 45401 and 45402, E. coli 44102, and C. albicans SC531 were spread on the surface of the LB agar plates. Aliquots $(200 \mu \mathrm{L})$ of the supernatant was loaded into an Oxford cup (outer diameter $=7.8 \pm 0.1 \mathrm{~mm}$, inner diameter $=6.0 \pm 0.1 \mathrm{~mm}$, and height $=10.0 \pm 0.1 \mathrm{~mm}$ ), which was placed on the surface of the agar. The size of the inhibition zone was measured until the formation of clear zone around the Oxford cup. The experiment was carried out in duplicates.

\section{Adherence of L. paracasei and B. longum to HT-29 Cells}

Adherence of L. paracasei and B. longum to HT-29 cells was examined as described previously (Zhang et al., 2010). The cells were cultured in RPMI-1640 medium (Hyclone, Logan, UT) supplemented with 10\% (vol/vol) fetal bovine serum (Hyclone) and incubated at $37^{\circ} \mathrm{C}$ in a $\mathrm{CO}_{2}$ incubator (HEPA class 100 , Thermo Fisher Scientific, Waltham, MA). The medium was changed daily until HT-29 monolayers were at 80 to 90\% confluence. The HT-29 monolayers prepared on glass coverslips and placed in 6-well plates (Corning Inc., Corning, NY) were washed twice with PBS buffer $(\mathrm{pH}$ 7.4). One milliliter of the bacterial cultures $(\sim 8$ $\log \mathrm{cfu} / \mathrm{mL}$ ) and $1 \mathrm{~mL}$ of cell line culture medium were added to each well of the tissue culture plate, and the plates were incubated at $37^{\circ} \mathrm{C}$ in $5 \% \mathrm{CO}_{2}$. After $2 \mathrm{~h}$ of incubation, monolayers were washed 4 times with sterile PBS followed by fixing cell lines with methanol for $30 \mathrm{~min}$. Nonadhering bacterial cells were washed away during this procedure. Gram staining was then carried out to stain the bacterial cells, which were examined under a microscope, then 100 HT-29 cells were randomly selected and the total number of adhering bacterial cells was counted as the adherence index. Each adherence assay was carried out in triplicate.

\section{Inhibition of Food-borne Pathogens Adhering to HT-29 Cells}

The ability of $L$. paracasei and B. longum to inhibit food-borne pathogens adhering to HT-29 cells was 
assayed according to the method reported previously (Zhang et al., 2010). Approximately $3 \times 10^{5} \mathrm{HT}-29$ cells per well were seeded in a 12-well plate (Corning Inc.). The medium was changed daily and HT-29 monolayers at 80 to $90 \%$ confluence were washed twice with PBS (pH 7.4) before conducting this experiment. In the exclusion assay, HT-29 monolayers were inoculated with $300 \mu \mathrm{L}$ of cell suspension of L. paracasei and B. longum $(\sim 7 \mathrm{log} \mathrm{cfu} /$ well) in RPMI-1640 medium and incubated for $1 \mathrm{~h}$ at $37^{\circ} \mathrm{C}$ in $5 \% \mathrm{CO}_{2}$. The HT-29 monolayers were then inoculated with $100 \mu \mathrm{L}$ of food-borne pathogens, respectively ( $\sim 7 \log \mathrm{cfu} /$ well), in RPMI-1640 medium and incubated for another $1 \mathrm{~h}$.

After incubation, nonadhering bacterial cells were removed by washing 4 times with sterile PBS, and cells then treated with $0.5 \mathrm{~mL}$ of $0.5 \%$ (vol/vol) Triton X-100 (Amresco, Solon, $\mathrm{OH}$ ) for $5 \mathrm{~min}$ in an ice-water bath. The mixtures were then serially diluted and plated onto LB agar plates for counting food-borne pathogens. As L. paracasei and B. longum were not able to grow in LB medium, the cell counts generated from LB agar plates were for pathogens. The ability of $L$. paracasei and $B$. longum to inhibit pathogens adhering to HT-29 cells was determined by comparing the number of pathogens adhering HT-29 cells in the presence or absence of $L$. paracasei or B. longum for the co-cultivation. Three independent experiments were carried out and each assay was performed in triplicate.

\section{Preparation of Fermented Milks Using L. paracasei, B. longum, and Viili Starter}

The viili starter was a generous gift from Cheng Luo (University of Tartu, Tartu, Estonia). To prepare various fermented milks, L. paracasei $(5 \%, \mathrm{vol} / \mathrm{vol}), B$. longum $(5 \%, \mathrm{vol} / \mathrm{vol})$, and viili starter $(5 \%, \mathrm{wt} / \mathrm{vol})$ were inoculated in $10 \%$ (wt/vol) in sterile skim milk and incubated at $25^{\circ} \mathrm{C}$ for $20 \mathrm{~h}$ followed by feeding these 3 fermented milks to mice. The fermented milks were kept in a refrigerator and were used within $1 \mathrm{wk}$ of preparation.

\section{Animal Experiment Design}

The animal experiment was approved by the Ethics Committee of Nanchang University (Jiangxi, China). Six- to 8-wk-old SPF BALB/c female mice (18 \pm 2 g) were purchased from the Hebei Center for Disease Control and Prevention (Hebei, China). After 1 wk of acclimatization in the Laboratory Animal Center at Nanchang University, the animals were randomly divided into 4 groups $(\mathrm{n}=3)$ and received 1 of the 4 fermented milks: (1) Bifidobacterium group (B. longum-fermented milk); (2) Lactobacillus group (L. paracasei-fermented milk); (3) viili group (viili starter-fermented milk); and (4) control group (sterile milk). The timeline of the animal experiment is shown in Figure 1.

Mice were gavaged with $1 \mathrm{~mL}$ of fermented milk or sterile milk each day during the second week. After that, each mouse was gavaged with $1 \mathrm{~mL}$ of $C$. albicans $(8 \log \mathrm{cfu} / \mathrm{mL}$ ) for $3 \mathrm{~d}$ in total, followed by a 7 -d recovery period. Feces were collected at d 0 (control stage) as a baseline, d 7 (after acclimatization), 14 (after feeding diets 1-4), 17 (after feeding C. albicans), 18 (1 d post- $C$. albicans infection), 21 (3 d post- $C$. albicans infection), and 24 (after recovery). Upon receipt of the feces, plate counting of each sample was carried immediately (Chen et al., 2014a).

\section{Enumeration of Fecal Microorganisms}

Microbial counts were enumerated according to previous studies (Chen et al., 2011b, 2014b). Briefly, serial dilutions of fecal samples were prepared in prereduced anaerobically sterilized (media) (PRAS) diluent, which were then plated on brain-heart-infusion agar plates,

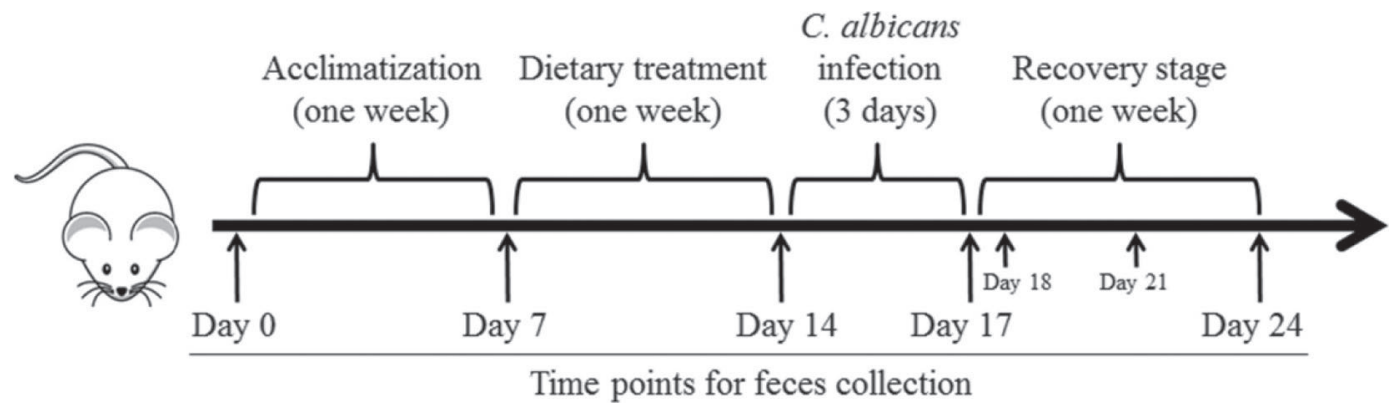

Figure 1. Animal experimental design. Time points (7 points) for collecting feces are indicated in the figure. Mice were divided into 4 groups and received 4 different diets: Bifidobacterium group (B. longum-fermented milk), Lactobacillus group (L. paracasei-fermented milk), viili group (viili starter-fermented milk), and a control group (sterile milk). Dietary treatments were carried out in mice in the morning, whereas the fecal sample collection was performed in the evening. 
MRS agar plates, or yeast extract-peptone-dextrose agar plates and then incubated anaerobically at $37^{\circ} \mathrm{C}$ for 24 to $36 \mathrm{~h}$ for counting colonies. Brain-heart-infusion agar, an enriched nonselective medium for the isolation and cultivation of most anaerobic microorganisms and other fastidious microorganisms, was used to count the total microorganisms in our study; MRS agar, an enriched selective medium intended for the isolation and cultivation of Lactobacillus, was used for counting gut lactobacilli. Yeast extract-peptone-dextrose agar was used for counting C. albicans.

\section{Statistical Analysis}

Data were analyzed and their means are presented in Figures 2 to 7 (SE measurement). Statistical analysis was performed in which ANOVA was calculated and the independent $t$-test was used to test for significance between groups $(P<0.05$ or $P<0.01)$. All analyses were carried out with using the statistics programs of SPSS 13.0 software (SPSS Inc., Chicago, IL).

\section{RESULTS}

\section{Acid Tolerance, Bile Salt Tolerance, and Antipathogenic Activity of L. paracasei FJ861111.1}

The populations of L. paracasei and B. longum after exposure to acid and bile salt are shown in Figure 2 and Figure 3. As shown in the figures, the suitable condition for both L. paracasei and B. longum was $\mathrm{pH} 7.0$ without

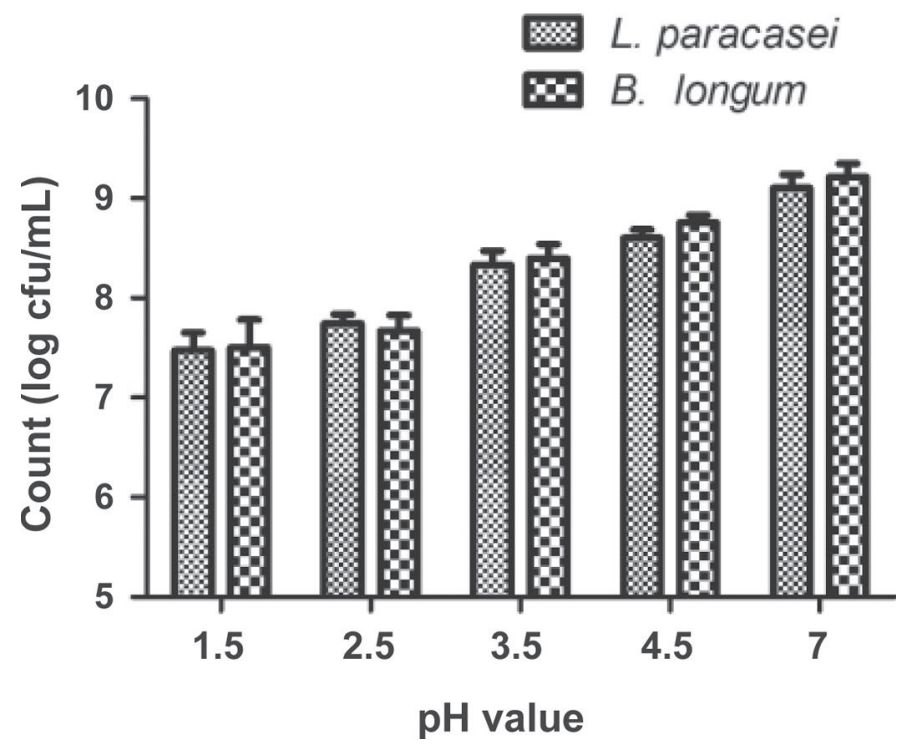

Figure 2. Tolerance of Lactobacillus paracasei FJ861111.1 and Bifidobacterium longum ATCC15708 to different acidic conditions. Error bars represent standard errors. bile salts supplemented to the medium. When $\mathrm{pH}$ was lowered and the bile salt concentration was increased, an obvious reduction in the viabilities of both $L . p a-$ racasei and $B$. longum was observed. In addition, the population of L. paracasei was $7.5 \log \mathrm{cfu} / \mathrm{mL}$ at $\mathrm{pH} 2.5$ and $6.0 \log \mathrm{cfu} / \mathrm{mL}$ in bile salt concentration of $0.5 \%$; values of $7.8 \log \mathrm{cfu} / \mathrm{mL}$ at $\mathrm{pH} 2.5$ and $6.0 \log \mathrm{cfu} /$ $\mathrm{mL}$ in bile salt concentration of $0.3 \%$ may suggest high survival of these organisms during passage through the gastrointestinal tract. Moreover, the viabilities of these 2 organisms under the same condition were not significantly different.

The antibacterial activity of $L$. paracasei and $B$. longum is shown in Figure 4. In general, the antibacterial activity of $L$. paracase $i$ was weaker than that of $B$. longum. It was found that $L$. paracasei could inhibit all the tested pathogens, especially Staph. aureus CMC (18 $\mathrm{mm}$ ) and Cr. sakazakii 45401 (16 $\mathrm{mm})$. This may be beneficial for food preservation and may likely kill the pathogens in the gut of the host.

\section{Adherence Capacity and Inhibition of Pathogens Adhering to HT-29 by L. paracasei FJ861111.1}

The adherence index of $L$. paracase $i$ and B. longum to HT-29 cell is shown in Figure 5. The adhered cells of $L$. paracasei to HT-29 cell were $1,173 \mathrm{cfu} / 100$ cells, which was similar to the adherence index of human-derived B. longum.

The result of inhibition of pathogens adhering to HT29 by $L$. paracasei and B. longum is shown in Figure 6. In general, L. paracasei could inhibit most of the tested pathogens adhering to HT-29 cells, including $S h$. dysenteriae 301 (49.5\%), Sh. dysenteriae 2457 (60.5\%), Staph. aureus Cowan1 (32.3\%), Staph. aureus CMC (3.2\%), Cr. sakazakii 45401 (21\%), Cr. sakazakii 45402 (24.7\%), E. coli 44102 (19.3\%), and C. albicans SC5314 (14.6\%). It appears that L. paracasei exhibited higher $(P<0.01)$ inhibition of Sh. dysenteriae adhering to intestinal epithelial cells than that of B. longum.

Counts of Total Bacteria, Lactobacillus, and C. albicans in Mice Before and After Feeding Fermented Milks and Before and After C. albicans Infection

The populations of total bacteria, Lactobacillus, and C. albicans cells in the feces of mice before and after $C$. albicans infection are shown in Figure 7. A significant increase was seen in the viable count of total bacteria $(P<0.01)$ at d 24 in both the Lactobacillus group and the viili group compared with that of the control group (Figure 7A). Little changes in the population of total bacteria were observed at d 24 among the Bifidobacterium, Lactobacillus, and viili groups compared with that 
at d 0 (Figure 7A). However, the administration of $C$. albicans had a significant reduction in the population of total bacteria in the control group. This suggests that $L$. paracasei, viili starter, and B. longum were able to sustain the population of total bacteria after the invasion of C. albicans.

Similarly, the population of Lactobacillus in Bifidobacterium, Lactobacillus, and viili groups were significantly $(P<0.01)$ improved at d 24 compared with that of control group (Figure 7B). Also, a significant reduction $(P<0.01)$ in the counts of gut Lactobacillus was noted in both the viili and control groups at d 24 compared with that at $\mathrm{d} 0$. This indicates less growthpromoting effect of viili starter-fermented milk than $L$. paracasei- and B. longum-fermented milk on the gut Lactobacillus in mice (Figure 7B).

Interestingly, it was found that all the fermented milk could inhibit the colonization of $C$. albicans in the gut of mice. This was evident by the significant $(P<0.01)$ reduction in the counts of $C$. albicans in the feces of mice at d 24 administrated with fermented milk (Figure 7C). Among the 3 fermented milks, L. paracasei-fermented milk exhibited the best scavenging ability to exclude the C. albicans cells, from 7.5 (in the control group) to $6.4 \mathrm{log} \mathrm{cfu} / \mathrm{mL}$ at d 24 (Figure $7 \mathrm{C}$ ).

\section{DISCUSSION}

Currently, most of studied probiotics, such as Lactobacillus rhamnosus GG and Lactobacillus casei strain Shirota, are from the L. casei group, including L. casei, L. paracasei, L. rhamnosus, and Lactobacillus zeae. It has been demonstrated that certain strains of $L$. paracasei exhibited important characteristics, for example good survival at low $\mathrm{pH}$, tolerance to bile salts, reducing serum cholesterol level, and improving sensory properties in fermented milk (Caridi, 2002; Chiang and Pan, 2012; Costa et al., 2014). In our previous study, $L$. paracasei FJ861111.1, isolated from viili, could acidify milk rapidly and produce a high quantity of exopolysaccharides (Chen et al., 2013). This suggests that this organism could be a potential functional starter for manufacturing fermented milks.

It is crucial for probiotics to deliver their beneficial effects on the host as living cells. Thus, tolerance to gastric acid and bile salts becomes important selection criterion for screening probiotic organisms (Jensen et al., 2012). It appears that L. paracasei FJ861111.1 showed a strong capability to survive in the acidic condition of $\mathrm{pH} 2.5$ and high bile salt concentration of $0.3 \%$; these conditions are similar in the stomach and small intestine of humans (Figure 2 and Figure 3). In addition, high viability of L. paracasei FJ861111.1 at $\mathrm{pH} 4.5$ suggests its high viable count during milk fermentation to deliver its beneficial effects to human upon consumption.

Adhesion to intestinal epithelial cells is another key criterion for probiotics so that they can colonize in the intestine for exerting their probiotic effects. Adhesion of pathogens allows the release of enzymes and toxins initiating necrotic processes directly into the target cell, thereby facilitating the invasion (Jankowska et al., 2008). A hidden but novel mechanism for probiotics to exclude pathogens from intestine is through the competition for adhesion sites on the surface of intestine and the production of antimicrobial components (Baccigalupi et al., 2005; Collado et al., 2006). Interestingly, a strong adherence of $L$. paracasei FJ861111.1 to HT-29 cells was observed when compared with human-derived B. longum ATCC15708 (Figure 5). This could be the base for L. paracasei FJ861111.1 to survive and colonize in human intestine to deliver its antiadhesion property against food-borne pathogens (Figure 4) and antibacterial activities (Figure 6). Thus, it was concluded that the viili-derived L. paracasei FJ861111.1 could be a good candidate as probiotics based on in vitro assays.

To examine in vivo anticolonization of pathogens by L. paracasei FJ861111.1, C. albicans was chosen for infecting mice due to inhibition of $C$. albicans adhering to HT-29 cell observed in the in vitro assay (Figure 6). Interestingly, this organism could significantly increase the population of total bacteria and Lactobacillus in the feces of mice post- $C$. albicans infection (d 24; Figure 3A and $3 \mathrm{~B}$ ). Moreover, the administration of $L$. paracasei FJ861111.1 before C. albicans infection was able to efficiently lower the counts of $C$. albicans in the feces of mice postinfection (Figure 7C). This suggests that this organism could exclude this pathogen from the gut of the host through its anticolonization ability.

Our intestinal tract is a nutrient-rich environment packed with up to 100 trillion microbes (Whitman et al., 1998). The diversity of microbiota in human gut is the result of co-evolution between microbiota and its hosts (Ley et al., 2006). It has been demonstrated that the imbalance of gut microbiota is closely associated with the development of specific diseases in certain host contexts (Ley et al., 2006). An examination of the viabilities of gut microbiota showed that the number of total bacteria and Lactobacillus in mice at d 17 post- $C$. albicans infection exhibited a little change compared with that at d 0,7 , and 14 before infection, whereas a significant reduction was observed in the control group compared with the Bifidobacterium, Lactobacillus, and viili groups (Figure 7A). In addition, a significant reduction in the counts of C. albicans (d 24) was noted; this contributed to the conclusion that L. paracasei 


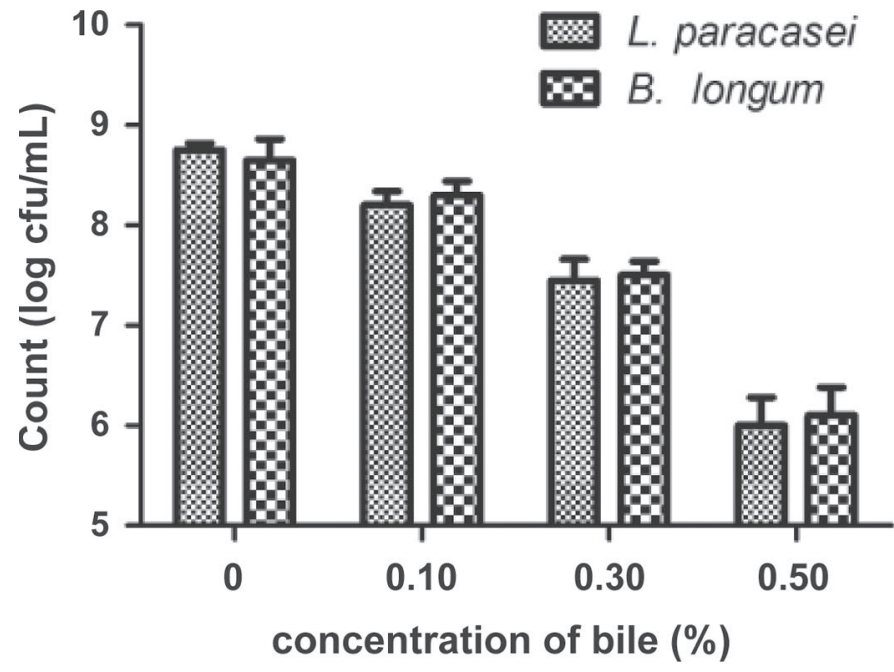

Figure 3. Tolerance of Lactobacillus paracasei FJ861111.1 and Bifidobacterium longum ATCC15708 to bile salts. Error bars represent standard errors.

FJ861111.1 was able to exclude pathogens from the gut and may regulate gut microbiota from imbalance to balance state.

\section{CONCLUSIONS}

Lactobacillus paracasei FJ861111.1 showed several important probiotic characteristics, including tolerance

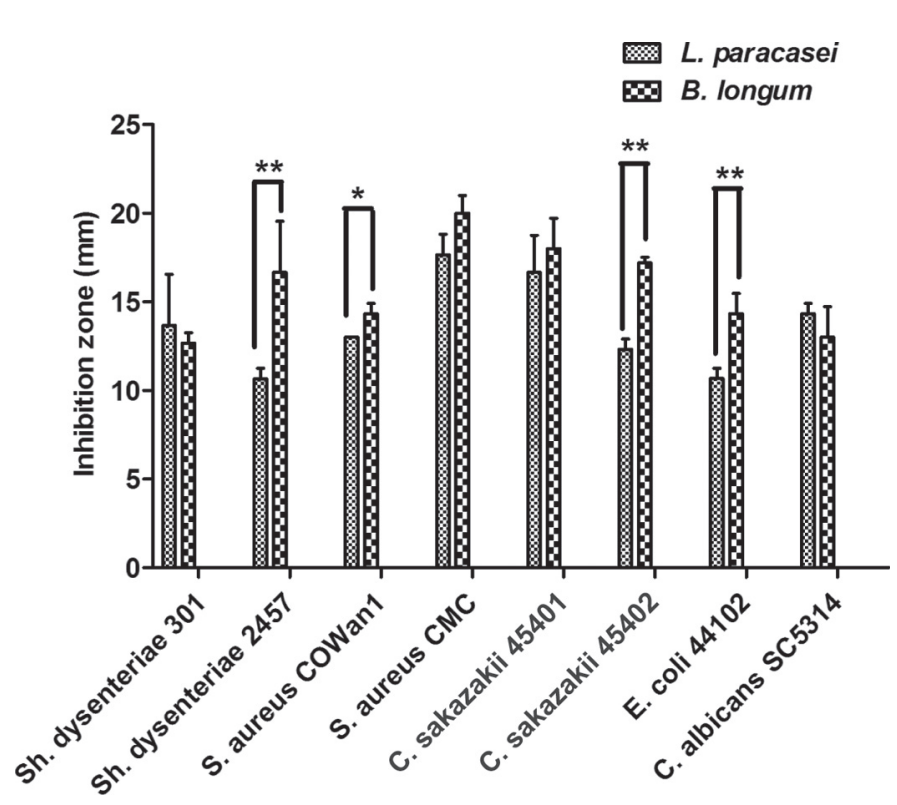

Figure 4. Antibacterial activities of Lactobacillus paracase FJ861111.1 and Bifidobacterium longum ATCC15708 against selected food-borne pathogens. The value of $L$. paracasei FJ861111.1 was compared with that of B. longum ATCC15708. Error bars represent standard errors. ${ }^{*} P<0.05 ;{ }^{* *} P<0.01$; all other values, $P>0.05$.

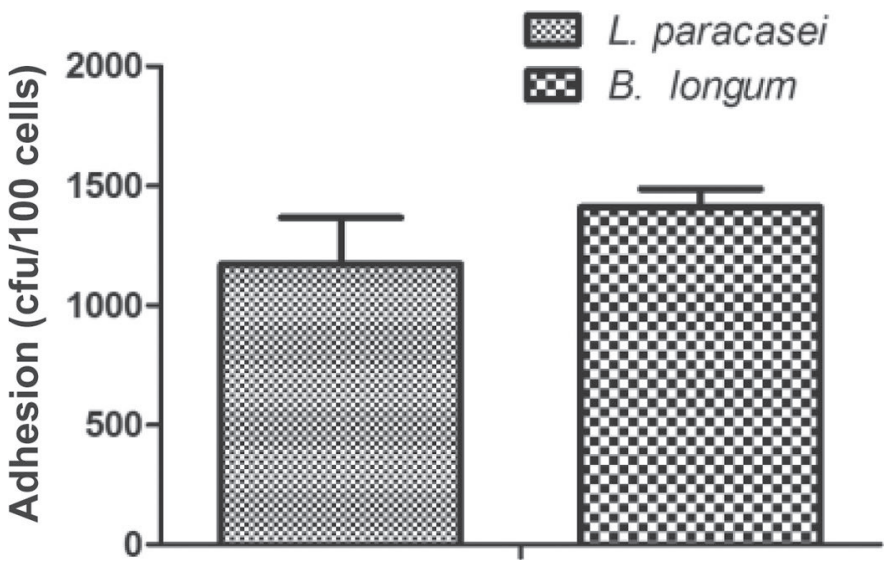

Figure 5. Adhesion capacity of Lactobacillus paracasei FJ861111.1 and Bifidobacterium longum ATCC15708 to HT-29 cells. Error bars represent standard errors.

to acid and bile salts, adhesion to HT-29 cells, antipathogenic activity, and inhibition of pathogens adhering to HT-29 cells. Consumption of L. paracasei FJ861111.1fermented milk increased the population of gut bacteria and Lactobacillus and reduced the counts of C. albicans in the feces of mice postinfection. However, no obvious changes in diversity of gut Lactobacillus in mice before and post-C. albicans infection was observed. We also concluded that L. paracasei FJ861111.1 could efficiently exclude pathogens from the gut postinfection.

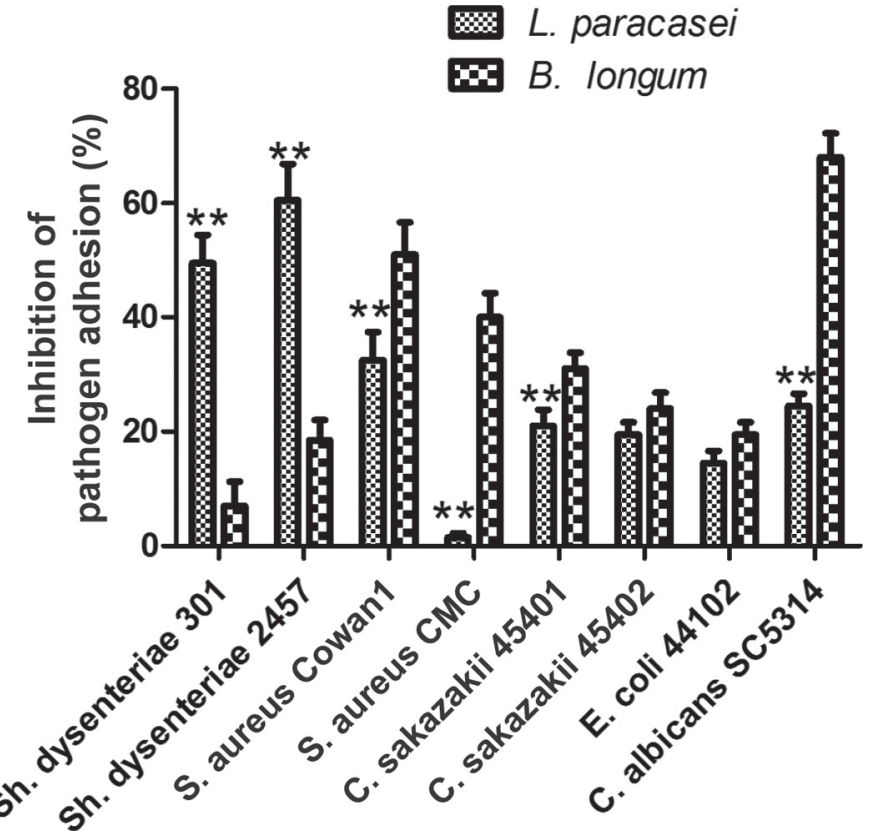

Figure 6. Inhibition of food-borne pathogens adhering to HT-29 cells by Lactobacillus paracasei FJ861111.1 and Bifidobacterium longum ATCC15708. Error bars represent standard errors. ${ }^{*} P<0.05$; ${ }^{* *} P$ $<0.01$; all other values, $P>0.05$. 


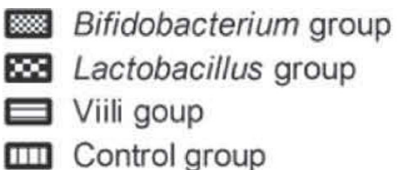

A

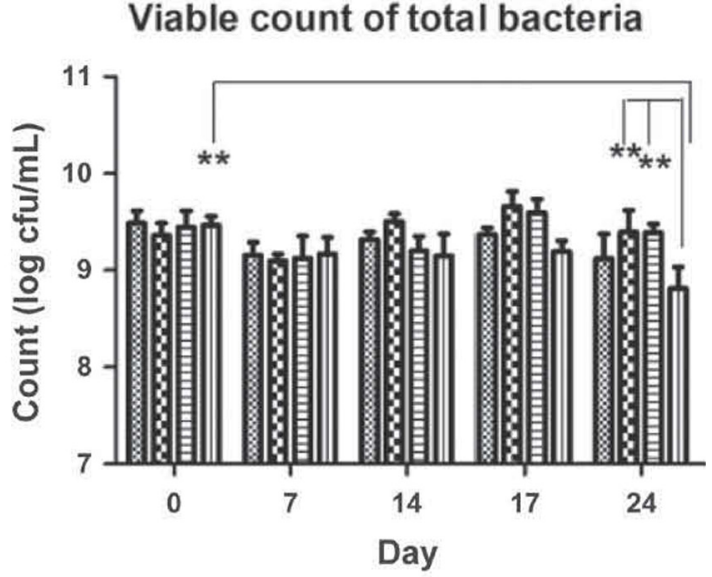

B

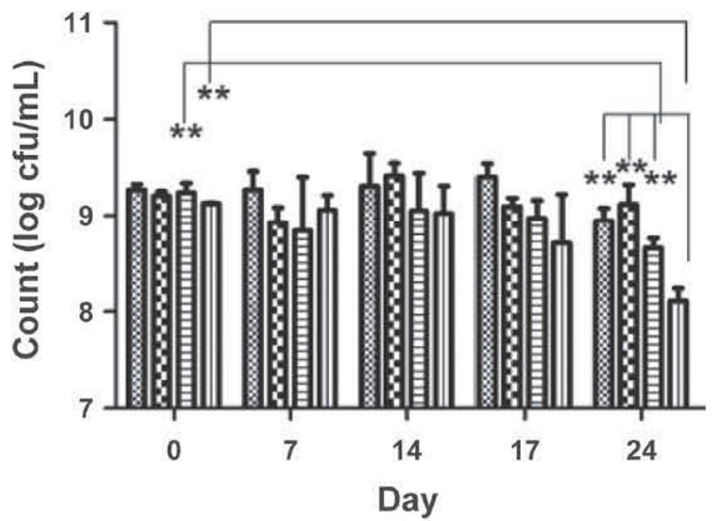

Viable count of C. albicans

C

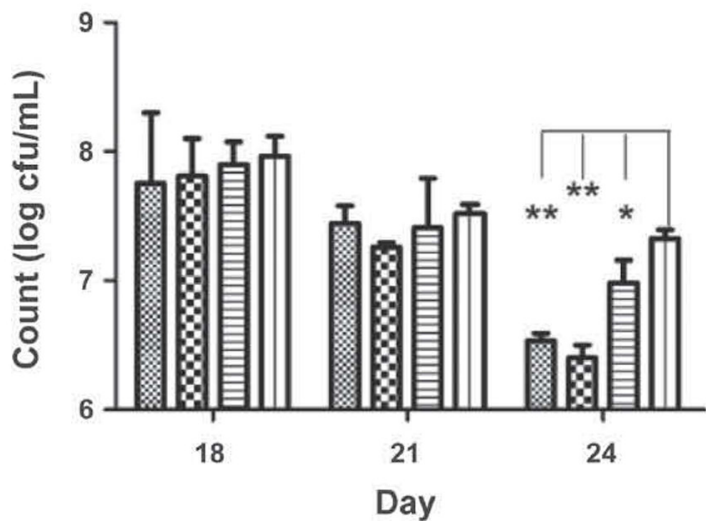

Figure 7. Viable counts of total bacteria (A), Lactobacillus (B), and Candida albicans $(\mathrm{C})$ in feces from mice before and post- $C$. albicans infection. Viable counts from Bifidobacterium group, Lactobacillus group, and viili group were compared with those with the control group. Error bars represent standard errors. ${ }^{*} P<0.05$; ${ }^{* *} P<0.01$; all other values, $P>0.05$.

\section{ACKNOWLEDGMENTS}

This work was supported by grants to Nanchang University (Jiangxi, China) from Jiangxi Province (grant no. 20142BAB215066, 20151BAB205001), Youth Science Funds of the Education Department of Jiangxi Province (grant no. GJJ14197), Jiangxi Province Health and Family Planning commission (2014A065), the Ministry of Science and Technology "Creation of Major New Drugs" special (2011ZX09102-009-01), the Landing Plan Project of Jiangxi Province (QT43-3), National Natural Science Foundation of China (21103159 and 21461015); and Science Foundation of Jiangxi Provincial Department of Education (KJLD14010).

\section{REFERENCES}

Alatossava, T., R. Li, and P. Munsch-Alatossava. 2013. From "viili" towards "termoviili", a novel type of fermented milk: Characterization of growth conditions and factors for a co-culture of Lactobacillus delbrueckii and Geotrichum candidum. J. Food Process. Beverages 1:8.

Baccigalupi, L., A. Di Donato, M. Parlato, D. Luongo, V. Carbone, M. Rossi, E. Ricca, and M. De Felice. 2005. Small surface-associated factors mediate adhesion of a food-isolated strain of Lactobacillus fermentum to Caco-2 cells. Res. Microbiol. 156:830-836.

Belkaid, Y., and T. W. Hand. 2014. Role of the microbiota in immunity and inflammation. Cell 157:121-141.

Botta, C., T. Langerholc, A. Cencic, and L. Cocolin. 2014. In vitro selection and characterization of new probiotic candidates from table olive microbiota. PLoS ONE 9:e94457.

Caridi, A. 2002. Selection of Escherichia coli-inhibiting strains of Lactobacillus paracasei ssp. paracasei. J. Ind. Microbiol. Biotechnol. 29:303-308.

Chae, J. P., V. Valeriano, G. B. Kim, and D. K. Kang. 2013. Molecular cloning, characterization and comparison of bile salt hydrolases from Lactobacillus johnsonii PF01. J. Appl. Microbiol. 114:121-133.

Chen, T., S. Li, and H. Wei. 2014a. Antibiotic resistance capability of cultured human colonic microbiota growing in a chemostat model. Appl. Biochem. Biotechnol. 173:765-774.

Chen, T., Q. Tan, M. Wang, S. Xiong, S. Jiang, Q. Wu, S. Li, C. Luo, and H. Wei. 2013. Identification of bacterial strains in villi by molecular taxonomy and their synergistic effects on milk curd and exopolysaccharides production. Afr. J. Biotechnol. 10:1696316968.

Chen, T., M. Wang, S. Jiang, S. Xiong, and H. Wei. 2011a. The application of polymerase chain reaction-denaturing gradient gel electrophoresis (PCR-DGGE) method in microbial screening. Afr. J. Biotechnol. 10:9387-9395.

Chen, T., Q. Wu, S. Li, S. Xiong, S. Jiang, Q. Tan, Z. Zhang, D. Zhu, and H. Wei. 2014b. Microbiological quality and characteristics of probiotic products in China. J. Sci. Food Agric. 94:131-138.

Chen, T., J. Yuan, X. Feng, H. Wei, and W. Hua. 2011b. Effects of enrofloxacin on the human intestinal microbiota in vitro. Int. J. Antimicrob. Agents 37:567-571.

Chiang, S.-S., and T.-M. Pan. 2012. Beneficial effects of Lactobacillus paracasei ssp. paracasei NTU 101 and its fermented products. Appl. Microbiol. Biotechnol. 93:903-916.

Collado, M. C., L. Jalonen, J. Meriluoto, and S. Salminen. 2006. Protection mechanism of probiotic combination against human pathogens: in vitro adhesion to human intestinal mucus. Asia Pac. J. Clin. Nutr. 15:570-575.

Costa, D. J., P. Marteau, M. Amouyal, L. Poulsen, E. Hamelmann, M. Cazaubiel, B. Housez, S. Leuillet, M. Stavnsbjerg, and P. Molimard. 2014. Efficacy and safety of the probiotic Lactobacillus 
paracasei LP-33 in allergic rhinitis: A double-blind, randomized, placebo-controlled trial (GA2LEN Study). Eur. J. Clin. Nutr. 68:602-607.

de Roock, S., M. van Elk, M. van Dijk, H. Timmerman, G. Rijkers, B. Prakken, M. Hoekstra, and I. de Kleer. 2010. Lactic acid bacteria differ in their ability to induce functional regulatory $\mathrm{T}$ cells in humans. Clin. Exp. Allergy 40:103-110.

Diplock, A. T., P. J. Aggett, M. Ashwell, F. Bornet, E. B. Fern, and M. B. Roberfroid. 1999. Scientific concepts in functional foods in Europe: Consensus document. Br. J. Nutr. 81(Suppl. 1):S1-S27.

Fung, T. C., D. Artis, and G. F. Sonnenberg. 2014. Anatomical localization of commensal bacteria in immune cell homeostasis and disease. Immunol. Rev. 260:35-49.

Gomes, A. M., and F. X. Malcata. 1999. Bifidobacterium spp. and Lactobacillus acidophilus: Biological, biochemical, technological and therapeutical properties relevant for use as probiotics. Trends Food Sci. Technol. 10:139-157.

Higgins, J. P., S. Higgins, A. Wolfenden, S. Henderson, A. TorresRodriguez, J. Vicente, B. Hargis, and G. Tellez. 2010. Effect of lactic acid bacteria probiotic culture treatment timing on Salmonella Enteritidis in neonatal broilers. Poult. Sci. 89:243-247.

Hill, C., F. Guarner, G. Reid, G. R. Gibson, D. J. Merenstein, B. Pot, L. Morelli, R. B. Canani, H. J. Flint, and S. Salminen. 2014. Expert consensus document: The International Scientific Association for Probiotics and Prebiotics consensus statement on the scope and appropriate use of the term probiotic. Nat. Rev. Gastroenterol. Hepatol. 11:506-514.

Jankowska, A., D. Laubitz, H. Antushevich, R. Zabielski, and E. Grzesiuk. 2008. Competition of Lactobacillus paracasei with Salmonella enterica for adhesion to Caco-2 cells. J. Biomed. Biotechnol. 2008:357964

Jensen, H., S. Grimmer, K. Naterstad, and L. Axelsson. 2012. In vitro testing of commercial and potential probiotic lactic acid bacteria. Int. J. Food Microbiol. 153:216-222.

Kitazawa, H., T. Toba, T. Itoh, N. Kumano, S. Adachi, and T. Yamaguchi. 1991. Antitumoral activity of slime-forming, encapsulated
Lactococcus lactis ssp. cremoris isolated from Scandinavian ropy sour milk, viili. Anim. Sci. Technol. (Japan) 62:277-283.

Lamari, F., K. Sadok, A. Bakhrouf, and F.-J. Gatesoupe. 2014. Selection of lactic acid bacteria as candidate probiotics and in vivo test on Artemia nauplii. Aquacult. Int. 22:699-709.

Ley, R. E., D. A. Peterson, and J. I. Gordon. 2006. Ecological and evolutionary forces shaping microbial diversity in the human intestine. Cell 124:837-848

Nakajima, H., Y. Suzuki, and T. Hirota. 1992. Cholesterol lowering activity of ropy fermented milk. J. Food Sci. 57:1327-1329.

Ranadheera, C. S., C. A. Evans, M. C. Adams, and S. K. Baines. 2014 Effect of dairy probiotic combinations on in vitro gastrointestinal tolerance, intestinal epithelial cell adhesion and cytokine secretion. J. Funct. Foods 8:18-25.

Wang, S. Y., H. C. Chen, J. R. Liu, Y. C. Lin, and M. J. Chen. 2008. Identification of yeasts and evaluation of their distribution in Taiwanese kefir and viili starters. J. Dairy Sci. 91:3798-3805.

Whitman, W. B., D. C. Coleman, and W. J. Wiebe. 1998. Prokaryotes: The unseen majority. Proc. Natl. Acad. Sci. USA 95:6578-6583.

Wood, B. 1998. Microbiology of Fermented Foods. Springer, Dordrecht, the Netherlands.

Wu, J., M. Li, L. Liu, Q. An, J. Zhang, J. Zhang, M. Li, W. Duan, D. Liu, and Z. Li. 2013. Nitric oxide and interleukins are involved in cell proliferation of RAW264.7 macrophages activated by viili exopolysaccharides. Inflammation 36:954-961.

Zhang, C., M. Zhang, X. Pang, Y. Zhao, L. Wang, and L. Zhao. 2012. Structural resilience of the gut microbiota in adult mice under high-fat dietary perturbations. ISME J. 6:1848-1857.

Zhang, Y. C., L. W. Zhang, Y. F. Tuo, C.-F. Guo, H.-X. Yi, J.-Y. Li, X. Han, and M. Du. 2010. Inhibition of Shigella sonnei adherence to HT-29 cells by lactobacilli from Chinese fermented food and preliminary characterization of S-layer protein involvement. Res. Microbiol. 161:667-672.

Zhao, L. 2010. Genomics: The tale of our other genome. Nature 465:879-880. 